\title{
El papel de una "apotencia" fundamental en la filosofía tardía de Schelling, y su influencia en la filosofía tardía de Heidegger
}

\section{(The role of a fundamental "lack of potency" in Schelling's later philosophy, and the influence on Heidegger's later philosophy)}

\author{
Alejandro ROJAS JIMÉNEZ
}

Recibido: 13 de junio de 2011

Aceptado: 20 de diciembre de 2011

\section{Resumen}

Este trabajo consta de dos partes. En primer lugar expondré la doctrina de las potencias de Schelling para mostrar en qué sentido esta doctrina se levanta sobre una apotencia, ein Potenzloses, de base. Posteriormente analizaré la influencia de esta apotencia en la Geviert de Heidegger.

Palabras clave: Das Potenzlose, doctrina de las Potencias, identidad, télos.

\section{Abstract}

This paper is divided into two main thematic blocks. First, it expounds the doctrine of the potencies of Schelling, trying to show how this doctrine is based in a Potenzlose. Secondly, I examine the influence of this Potenzlose to Heidegger's Geviert.

Keywords: Das Potenzlose, doctrine of the potencies, idenitity, télos. 
„La liberté souveraine du Créateur est sauvegardée par la même processus de recel et de retrait. Dieu reste inexorablement le Dieu caché, le Potenzloser. Il se dissimule derrière les puissances qu'Il a distendues et extraverties, sa divinité comme actus purissimus demeure intangible, Il n'entre pas dans le procès cosmique."

\section{Introducción}

El intento de Schelling por entender el absoluto a partir de causas internas lo conducen hacia la noción de potencia. La primera vez que emplea la noción de potencia es en el año 1798, en Von der Weltseele. Si bien, no se desarrolla más allá de la Natürphilosophie hasta 1801 con Darstellung meines Systems der Philosophie. Desde este momento la identidad absoluta $\mathrm{A}=\mathrm{A}^{2}$ es pensada en función de un método de preponderancia parcial según el cual nos encontramos con un predominio objetivo $(\mathrm{A}=\mathrm{B}+)$ o un predominio subjetivo $(+\mathrm{A}=\mathrm{B})$. Gracias al método de la preponderancia parcial puede Schelling pensar el absoluto, si bien lejos queda una visión armónica de esta dualidad. En su lugar, el movimiento y la oposición, así como ocurriera en la filosofía de Anaximandro o Heráclito (o Aristóteles ${ }^{3}$ ), se consolidan como palabras fundamentales de una filosofía que afirma un proceso en el que el absoluto siempre se oculta como tal tras cada determinación en la que se expresa, desapareciendo toda perspectiva de alcanzar un último momento del proceso en el que se resuelvan las diferencias.

Puede parecer que el hecho de que no haya un momento último contradice la finalidad pretendida de expresión del absoluto. Y sin embargo, que no haya un final no significa que no pueda haber una finalidad. En este caso concreto lo que nos encontramos es que la finalidad de la determinabilidad (productividad) es su expresión mediante la determinación (producto) aún cuando al mismo tiempo, en la medida en que la determinabilidad no puede ser inmediatamente manifiesta, no se apunta a ninguna identificación final. La determinabiliad o productividad se expresa en el producto, "finitizándose" (sich verendlichen), a la par que se oculta en un constante Reproduciertwerden ${ }^{4}$ que no tiene ningún final debido a la infinitud de la indeterminabilidad del ser absoluto. Y por ello "finitizarse" significa al mismo tiempo liberación de toda forma finita de la realización de tal modo que queda libre para

\footnotetext{
1 Tilliette (1987), p. 132.

2 „Ideales und Reales bilden zwar im endlichen Sein eine Einheit, aber so, dass immer eines von beiden überwiegt. In der Natur überwiegt das Objektive, in der Welt des Geistes das Subjektive, jeweils in verschiedenen Stufungen. Schelling hat diese ontologischen Grundverhältnisse in einem Liniengleichnis veranschaulicht (IV, 137; VII, 184).“ Hennigfeld (2001), p. 21.

3 Cfr., Phys., 260a ss.

4 Einl., SW I/III, p. 315.
} 
cualquier determinación: frei vom aller Bestimmtheit y al mismo tiempo y precisamente por ello frei zu aller Bestimmtheit ${ }^{5}$.

La productividad incondicional del ser absoluto "se oculta detrás de los fenómenos individuales en los que se hace manifiesta" 6 . Ella no puede ser pensada sin relación a lo péras, puesto que la "productividad incondicional no llega a ser manifiesta de un modo inmediato"7. Los conceptos de ser o absoluto (determinabilidad) y de forma (determinación), se pertenecen mutuamente, tanto que ninguno puede ser pensado sin el otro. Fue en el Filebo de Platón donde se encontró Schelling con las nociones de determinabilidad y límite 8 , de modo que bien podría Schelling haber aprendido en el Filebo esta relación.

Las formas diferencian el ser, es decir determinan el ser al tiempo que él comparece en ellas. El absoluto comparece siempre bajo distintas figuras en una sucesión que no encuentra fin ${ }^{9}$, pero donde observamos una clara finalidad (télos): la manifestación del ser absoluto en cada determinación. La meta de la productividad son los productos, aunque la determinabilidad permanezca libre de toda determinación, y una liberación respecto de las formas limitadas se eternice (perpetuitas vitae). Así, vemos una afirmación del tiempo como continuidad o constancia por razón de una libertad que lo es para cualquier determinación. Como dice Heidegger en el año 1941, encontramos aquí una "constancia no afectada por la sucesión"10. Pero no porque el ser absoluto sea un Weiter-Sein, sino porque la indiferencia entre la determinabilidad y la determinación permanece eternamente. Por ello propondría más bien la siguiente expresión para el ser: das Zusätzlich-Sein. Eternamente significa aquí sempiternitas in agendo. Una vita sempiterna del ser en la que se reproduce constantemente la misma estructura: "la distinción de forma y ser no anula el ser, sino que más bien lo afirma como aquella identidad que permite entender las formas como tales formas de eso mismo idéntico. Por eso aplica Schelling esta distinción nuevamente a cada nivel de reflexión: por ejemplo, el ser absoluto aparece en las formas de la naturaleza y el espíritu, el ser de la naturaleza y el espíritu en las formas de lo orgánico y lo inorgánico, el ser de lo orgánico en las formas de las plantas y los animales, etc."11.

\footnotetext{
5 Cfr., Halfwassen (2010), p. 76.

6 VW, SW I/II, p. 382.

7 Jacobs (2004), p. 92.

8 Cfr., Jacobs (2011a), p. 89.

9 „Kontinuität ist jedoch der beste Name, um diesem Signifikanten der Zeit zu bescreiben, in den sich die Dinge einzuschreiben scheinen. Und außerdem begründet diese Kontinuität auch den Vorgang, um die Differenz transzendental/empirisch zu tilgen, die sich gerade ausgehend von dieser Kontinuität auflöst.“ Leyte (2010), p. 152.

10 Heidegger (1971), p. 208.

11 Jacobs (2011a), p. 89
} 


\section{La doctrina de las potencias}

La búsqueda del absoluto12, que Schelling emprende de la mano de Kant y Platón, lo conduce más allá de Fichte. Su Darstellung meines Systems der Philosophie (1801), junto con Bruno (1802) y las Vorlesungen über die Methode des akademischen Studium (1803) constituyen una clara filosofía propia: la Identitätsphilosophie, según la cual el absoluto llega a ser contemplado con ayuda de la doctrina de las potencias. Se trata de una nueva representación del absoluto que se orienta en un nuevo método: der Methode des Potenzierens. La idea germen de la doctrina de las potencias la ha formulado con éxito Falgueras como sigue: "no hay naturaleza sin espíritu, ni espíritu sin naturaleza, no hay producción sin saber ni saber sin producción, aunque naturaleza y espíritu, producción y saber, sean contrarios. Como ambos no pueden estar íntegramente a la vez, pero tampoco por separado, la única posibilidad que queda es la de que estén a la vez procesualmente, es decir, con preponderancia parcial, en dos procesos paralelos o separados, pero armónicos"13.

Ahora bien, un momento decisivo en la evolución de esta doctrina cambiará su formulación inicial fundamentalmente. Cuando Schelling persigue salir al paso de las críticas acerca de la relación entre necesidad y libertad, fija un infundamento (Ungrund) que va a dotar de una nueva solidez y consistencia aquella Potenzenlehre: cuando Schelling intenta responder a la pregunta de por qué la representación panteísta del absoluto concentra la más alta libertad y la más alta necesi$\mathrm{dad}^{14}$, se percata de que la doctrina de las potencias debe reposar sobre una apotencia sin la cual se disolvería todo ser y todo equilibrio de preponderancia parcial.

La noción de apotencia apunta a un ser retraído (ein Gehemmtsein) que quiere permanecer en el misterio y que se distingue respecto de las posibilidades de las potencias (Duplizität). La apotencia o Potenzlose está vinculada a la noción de infundamento, el cual viene a ocupar a partir del escrito sobre la libertad de 1809 el lugar que desde 1801 había ocupado la identidad del absoluto como principio del sistema. El infundamento no designa más aquella identidad. Y no porque nombre una impotencia, sino porque designa un nuevo sentido de la representación de la revelación de la determinabilidad que no tiene nada que ver con el absoluto hegeliano pensado como totalidad de las determinaciones ${ }^{15}$. En la dirección de esta

12 "La genèse historique se récapitule dans un système qui veut être déroulé comme un devenir. Les époques de la pensé se reproduisent en moments du système, l'évolution singulière se hausse à l'universel." Tilliette (1992), p. 504.

13 Falgueras (1999), p. 253.

14 „Nothwendigkeit und Freiheit stehen ineinander, als Ein Wesen, das nur von verschiedenen Seiten betrachtet als das eine oder andere erscheint, an sich Freiheit, formell Nothwendigkeit ist." PhU, SW I/VII, p. 385 .

15 „Hegels Gedanke, dass sich Gott im Menschen verwirklicht, ist die höchste Negativität, die höchs- 
representación comparece una indiferencia respecto de una determinación absoluta, porque la libre productividad incondicional tiene que ser pensada libre para cualquier determinación, y de este modo tiene que ser entendida en proceso, como el "eterno hecho de la autorevelación"16: el tiempo de la autogeneración de la historia de la revelación. Por eso no se trata más de potencias, tampoco de impotencia alguna, sino de una apotencia: una indiferencia respecto de la totalidad de las determinaciones, en función de la cual el tiempo es concebido como "aquel momento de la decisión, donde la voluntad originaria sale de la eternidad de su puro poder ser para revelar su grandeza según el tiempo"17.

La noción de indiferencia es polémica. Por un lado puede significar desinterés y por otro indiferenciación. El problema reside en que no podemos limitarnos a entender la indiferencia bajo uno de los aspectos, sino que es preciso distinguirlos ya que los dos juegan un papel importante en la filosofía de Schelling. En unos casos la indiferencia designa la indiferenciación de los principios ante omen affirmationen et negationen, en otros casos el desinterés respecto de un producto que sea la productividad misma.

Schelling intenta pensar el infundamento absoluto como indiferenciación: lo real y lo ideal (oscuridad y luz) son representados como no opuestos, pues se trata de una dualidad donde aún no hay oposición. Es la absoluta falta de diferencia. Si no fuera el ser ante omnem affirmationem et negationem estaría al final de la sucesión. Pero no está al final18, sino que está ya en cada producto, porque el ser es la realidad del producto posible.

El ser puede ser Eins von Allem ${ }^{19}$ porque no está al final, sino ante. La indiferenciación no es pensada como la absoluta identidad entre determinabilidad y determinación que precedería a ambas. La indiferenciación de los principios no puede ser tomada bajo un pensamiento que fuera un producto. Ella es más bien lo inconcebible que está en cada producto. Y esta no producción de la productividad en un producto no es entendida como una impotencia, sino como un desinterés o indiferencia: lo que la productividad quiere es producir algo. Este desinterés trastoca de un modo fundamental la doctrina de las potencias. Lo que debe ser explicado en dos pasos. En primer lugar debe ser comentado el origen especulativo del cambio. En segundo lugar debe ser aclarado con mayor precisión lo que designa la noción de apotencia.

te Unangemessenheit zum Wesen der Gottheit.“ Koslowski (2001), p. 724.

16 „Nach der ewige Tat der Selbstoffenbarung ist nämlich in der Welt, wie wir jetzt erblicken, alles Regel, Ordnung und Form; aber immer liegt noch im Grunde das Regellose, als könnte es einmal wieder durchbrechen, und nirgends scheint es, als wäre ein anfängliches Regelloses zur Ordnung gebracht worden." PhU, SW I/VII, 359, p. 360.

17 Friedrich (2009), p. 192.

18 Cfr., WA, SW I/VIII, p. 232.

19 Cfr., Buchheim (1992). 


\subsection{El origen especulativo del cambio de la Potenzenlehre: el fracaso de la identidad mediada}

El origen especulativo del cambio de la doctrina de las potencias reposa en el fracaso de la identidad mediada. La identidad mediada fue traída para entender la espontaneidad. La espontaneidad como noción para el fundamento nombra un movens per se como causa sufficiens omnis actus sui. Continuar la cuestión hacia el fundamento de la voluntad carece de sentido: aquí estamos sencillamente ante el punto último de la cuestión ${ }^{20}$. La espontaneidad tiene su origen en el voluntarismo medieval (Scoto y Ockham). Fue la noción de fundamento también de la edad moderna. Puede aclarar el paso de la potencia al acto sin hablar de ninguna suerte de causa final, en tanto que la existencia del infinito, omnipotente, es para el nominalismo lo eterno bajo toda verdad de donde emerge cualquier verdad que nuestro entendimiento pueda captar ${ }^{21}$. Lo que Dios quiere, es por eso bueno y verdadero.

Aunque la espontaneidad y la infinitud surgen a propósito de una discusión escotista acerca del fundamento, Ockham afirma que la causa eficiente no puede ser entendida: si el fundamento no tiene fundamento, parece que entonces no hay nada que entender. Pero podemos pensar que sería posible entender este fundamento: la causa puede ser conocida siempre que se observe lo que ha causado. Esto es, si se sigue al Cusano 22 y si se retoma la estructura $A$ efficit ut $A$ sit totum. Falgueras afirma que la idea fundamental del proyecto unitario del idealismo alemán es que "la realidad absoluta puede ser considerada igual que la intelección, como un proyecto expresivo que va necesariamente de lo implícito eficiente a lo explícito formal. En ambos términos del proceso se contiene la totalidad de distinta manera" 23 .

Implicitus-efficiens nombra la totalidad sintéticamente, mientras que explicitusformalis nombra la totalidad analíticamente. Si realmente esto fuera correcto podríamos decir que el idealismo es una suerte de spinozismo24. Esta consecuencia es obviamente problemática, ya que el idealismo alemán es más amplio que el spinozismo. Sin embargo, este bosquejo del proyecto del idealismo alemán nos permite representarnos con claridad el origen especulativo del cambio que sufre la doctrina de las potencias de Schelling: contra este propósito de la filosofía de la identidad

\footnotetext{
20 „Vielmehr bestimmt der Wille selbst sich ganz allein und ist das movens per se in jeder Handlung, die einzige und totale Ursache seiner Volitionen, causa sufficiens omnis actus sui. Die weitere Frage nach dem Grunde noch des Willens ist sinnlos: hier ist ein schlechthin Letztes." Heimsoeth (1958), p. 220.

21 Cfr., ibid., p. 233 ss.

22 Cfr. García (2007), p. 8.

23 Cfr., Falgueras (1976), p. 145.

24 "Naturalmente, las diferencias entre el sistema de Espinosa y el de Schelling son grandes desde el punto de vista de los contenidos, pero no desde el punto de vista funcional del hallazgo especulativo, que es común a todas las filosofías del absoluto.” ibid, p. 147.
} 
trae la filosofía tardía de Schelling un absoluto que no puede ser ya más pensado según el proceso de autoconstitución de la identidad mediada.

A partir de 1809 Schelling se percata de que la vermittelte Identität no sirve para entender la espontaneidad como fundamento 25 , porque el ser no entra completamente en el proceso. Quiero decir, Schelling señala un nuevo momento, un momento inicial y originario que no se debe buscar en la identidad absoluta, sino en la oscuridad del abismo. Por esta oscuridad des Abgrundes acaba siendo la filosofía una suerte de acción libre del espíritu ${ }^{26}$, de tal modo que la filosofía de Schelling se muestra cada vez más como el inicio de un nuevo modo de filosofar27: una filosofía, post-idealista' 28 que tiene como consecuencia que Schelling se vea forzado a prescindir de todo lo finito 29 .

\subsection{La apotencia y la filosofía que prescinde de todo lo finitio}

\subsubsection{La indiferenciación de los principios}

Las philosophischen Untersuchungen constituyen un tratado en el que se realiza la distinción fundamental de dos principios, a saber: entre el ser en tanto que fundamento y en tanto que existe. Estos principios están indisolubles en Dios. No así en el hombre ${ }^{30}$. La posibilidad de la desvinculación de aquellas fuerzas vivas (Band von lebendigen Kräften) es la posibilidad del mal como desarmonía ${ }^{31}$. Si no se diera la posibilidad del mal (distinguiendo ésta de su realidad), no se daría tampoco libertad alguna, porque la libertad implica la posibilidad de decidir, y por ello una capacidad para el bien y el mal32.

La armonía está pensada aquí como una unidad de los diferentes principios donde uno no puede ser por sí ni tampoco sin el otro33. Se pertenecen mutuamente,

25 „Es ist Schellings Freiheitslehre der philosophischen Untersuchungen, mit der in der Geschichte des deutschen Idealismus dadurch ein Neuanfang gemacht wird, da $\beta$ das Verhältnis von Spontaneität und Notwendigkeit einer Deutung unterzogen wird, die an deren Gegensatz festhält und ihm nicht zum Verschwinden bringt." Sturma (1995), p. 154.

26 EV, SW I/ IX, p. 229.

27 Cfr., Duque (1998b), p. 54.

28 "el último Schelling lleva a su culminación el Idealismo y, de este modo, lo hace naturalmente desembocar en el ancho piélago de la filosofía contemporánea." Duque (1998a), p. 928.

29 „Hier muss alles Endliche, alles, was noch ein Seyendes ist, verlassen werden, die letzte Anhänglichkeit schwinden; hier gilt es alles zu lassen -nicht bloß, wie man zu reden pflegt, Weib und Kind, sondern was nur ist, selbst Gott, denn auch Gott ist auf diesem Standpunkt nun rein Seyendes“ EV, SW I/IX, p 217.

$30 \mathrm{PhU}, \mathrm{SW}$ I/VII, p. 365.

31 Cfr., ibid., p. 390.

32 Cfr., ibid.., p. 352.

33 Cfr., ibid., p. 408. 
como un ser que sólo es considerado desde los distintos frentes cuando comparece uno u otro. Este ser sólo se divide para que cada uno de los aspectos, que no pueden darse simultáneamente, puedan llegar a ser, a través del amor (el eterno vínculo entre ambos), uno.

Schelling emplea distintas nociones para designar el primer principio, esto es el fundamento de la existencia. Así por ejemplo, habla de la naturaleza en Dios. Entiende este principio como el afán irracional de una voluntad ciega que de forma natural aspira a desplegarse ${ }^{34}$. El fundamento es pensado aquí pues como principio oscuro e irracional: la voluntad de fundamento quiere, sin poder elegir racionalmente o conscientemente. No puede por eso realizarse ni encontrar paz. Es un fuego devorador. Para ser voluntad racional debe determinarse.

Porque esta ansia sola no produce nada, se encuentra este principio eternamente frente a otro: si el primer principio o potencia es pura autoreferencialidad, Selbstbezüglichkeit, el segundo es una universalización, Universalisierung, que procede de sí mismo. El nombre de Schelling para esto es lógos. En virtud de esta procedencia de sí (Hervorgang aus sich selbst) hablamos de una autodeterminación libre, eine freiwillige Selbstbestimmung 35 . Mediante ambos principios puede ser pensada la libertad.

Claro que la autodeterminación no puede, sin embargo, ser pensada sin una meta. Y por eso está obligado el pensamiento acerca de la libertad a afirmar un tercer y último principio que designa aquello que debe ser, esto es, la armonía de los principios o amor. Los tres principios o potencias son así pensados en una unidad.

„El amor, que antecede a la dualidad de fundamento y existencia, Schelling lo nombra infunamento" 36 . Si el infundamento nombra un ser que antecede a toda dualidad, se concluye entonces que el infundamento nombra una finalidad (Zweck): el infundamento se divide en dos inicios igualmente eternos sólo para que los dos, que no pueden darse por separado, a través del amor, sean uno ${ }^{37}$.Esta distinción nombra por un lado Dios como absoluto (sofern es existiert) y por otro lado la naturaleza en Dios (Grund von Existenz). Naturaleza significa aquí Hervorbringen, es decir, crear o producir, pero de tal modo que lo producido es la propia revelación de Dios. Esto quiere decir, que la consecuencia tiene que tener vida por sí. Tenemos que decir que, si los productos son expresión de Dios, ellos tienen que ser productivos, esto es, tienen que estar vivos. Para Schelling lo producido es efectivamente un producto, pero distingue la producción mecánica, das mechanisch Produzierte, del organismo producido, vom organisch Hervorgebrachten.

\footnotetext{
34 Cfr., ibid., p. 376.

35 Cfr., SPV, I/VII, p. 430.

36 Hennigfeld (2001), p. 127.

37 „Der Ungrund teilt sich aber in die zwei gleich ewigen Anfänge, nur damit die zwei, die in ihm, als Ungrund, nicht zugleich oder Eines sein konnten durch Liebe eins werden.“ PhU, SW I/VII, p. 408.
} 
La producción mecánica no da como resultado algo vivo, sino un producto muerto. Pero el organismo producido es por el contrario una "criatura" que tiene libertad ella misma para seguir ejerciendo y actuando. Como quiera que aquí lo distintivo es distinguir modos de producir, hacemos bien en distinguir el engendrar, das Zeugen, y el mero hacer algo donde lo hecho no tiene vida por sí, dem Bewirken. Nosotros debemos considerar la sustancia indeterminada a partir de sus consecuencias, pensarla como una única sustancia que se expresa. Se trata en ambos casos de un mismo ser considerado desde dos puntos de vista distintos, y que no pueden ser pensados uno sin el otro: porque la determinabilidad de la pura Wesenheit nunca puede ser manifiesta inmediatamente. Y así encontramos aquí el misterio del amor ${ }^{38}$. Esta unión deja perdurar sin embargo la diferenciación de los principios ${ }^{39}$, por eso no nombra esta finalidad ningún fin del proceso. Se trata aquí, en palabras de la tradición, de la omnipotencia de Dios que no encuentra ninguna limitación. Esta omnipotencia es pensada en relación a una "apotencia" fundamental, ya que si la productividad se crease entonces no sería infinita: "si se le intenta dar la vuelta, pensar un producto en el que no hubiera ya nada productivo, significaría llevar la productividad a su fin" 40 . Esto no significa en absoluto que la productividad no se manifieste en los productos, sino que la productividad se manifiesta en ellos al tiempo que se oculta.

Porque no se pretende la superación de los principios, digo apotencia en lugar de impotencia, como también hace Schelling en las Weltaltern ${ }^{41}$, para indicar que no se trata de buscar una determinación. Precisamente porque el ser libre es libre de toda determinación puede ser libre para toda determinación, y siempre que el ser absoluto declina una forma limitada, muestra en este movimiento de liberación su infinitud. Las posibilidades infinitas del Sich-verwirklichen-Könnens se diferencian de la realidad del ser originario. Si "realizarse" significa como se dijo "finitizarse", no puede esta limitación del ser encontrar calma alguna, porque el puro ser mismo no puede ser realizado en ninguna de estas "finitizaciones", ya que, como se ha dicho, la Wesenheit no puede realizarse como tal. No podemos entonces hablar de ningún fin en el sentido de Ende. Sí de télos o Zweck: la voluntad originaria expresa su libertad y productividad (realidad) por mediación de los productos (posibilidades), sólo que no por mediación de un producto. Una ,dialectique de la révélation et du recel " 42 puede salvaguardar esta libertad. Por el contrario, la identidad de los principios impediría esta revelación de la determinabilidad.

\footnotetext{
38, ,...) das Geheimnis der Liebe, dass sie solche verbindet, deren jedes für sich sein könnte und doch nicht ist, und nicht sein kann ohne das andre." ibid., p. 408.

39 ,anstatt also, daß dieser die Unterscheidung wieder aufhöbe, wie gemeint wurde, setzt und bestätigt er sie vielmehr.“ ibid., p. 407

40 Jacobs (1999), p. 48.

41 ,vielmehr es ist außer und über aller Potenz, das an sich Potenzlose.“ WA, SW I/VIII, 234.

42 Tilliete (1987), p. 132.
} 


\subsubsection{La dialéctica de la revelación y la ocultación}

Una finalidad indeterminada no es pensable. Pero esto no significa que tengamos que pensar un Ende. Schelling sabía que apeiron y péras se hallan uno junto al otro 43 . Se habla de apeiron como tal en tanto que se distingue de péras, pero apeiron (determinabilidad) y péras (determinación) permanecen uno junto al otro de tal modo que ninguno puede ser pensado sin el otro.

Lo que nos encontramos es el descubrimiento de la necesidad de colocar una limitación: sólo si la voluntad se autolimita ${ }^{44}$ puede comparecer. La voluntad del fundamento no puede encontrar nunca calma, porque es un fuego devorador. Por ello, para ser voluntad racional, debe autodeterminarse. Se determina para poder comparecer. Ahora bien, la voluntad libre quiere su determinación, pero no su fin o su límite. Por eso no podemos pensar esta determinación como fin ${ }^{45}$, sino que debemos pensarla como una dimensión del télos.

En oposición al fin, el cual conlleva la disolución de los principios, observamos un movimiento en el que el ser indeterminado que determina el ser (autodeterminación) aparece, y que además diferencia al mismo tiempo la determinación respecto del ser indeterminado. Siempre permanece la constancia de la productividad bajo nuevas figuras ${ }^{46}$. El ser aparece bajo distintas figuras en una sucesión que aunque no encuentra fin, encuentra sin embargo una clara meta (télos): la aparición del ser absoluto en los productos respecto de los cuales es libre, de modo que queda libre a su vez para toda determinación. Por eso se puede hablar de una liberación ${ }^{47}$ respecto de cada forma limitada. Observamos aquí una dialéctica de la revelación y la ocultación. El movimiento no es ningún proceso ilimitado sin sentido: consiste en una liberación. Es el en dónde donde se halla la libertad.

No se trata de pensar la determinación sin meta, sino de no pensar dicha meta como un límite del querer. A télos pertenece lo péras como determinación conceptual, pero no como límite del querer. ¿Qué puede significar meta si no designa ningún fin? Respuesta: libertad. Si no se concibe que la verdadera meta es la libertad, adviene entonces un proceso ilimitado. Pero no está en éste la meta, sino en la liber$\operatorname{tad}^{48}$.

${ }^{43}$ Schelling (1794), p. 70.

${ }^{44}$ Cfr., SPV, SW I/II, p. 430.

${ }^{45}$ Cfr., GPPh, p. 95.

46 "El curso del tiempo, en su incesante seguir, es inflexible, siempre lo mismo, constante. La continuidad del tiempo acaba con toda la soberbia de lo estable, de lo que aspira a ser de un modo fijo; y ella misma es ahora lo único realmente existente y permanente, lo redondo e igual a sí mismo, lo que eternamente retorna y se mantiene." García (1999), p. 123.

47 „Die Wirklichkeit der Freiheit muss als das Geschehen der Befreiung gedacht werden.“ Hutter (1996), p. 182.

48 Cfr., Conferencia de Jacobs en la Universidad de Málaga en noviembre de 2010. Esta misma idea 
El fin no es ningún objeto del querer, del mismo modo que decimos por ejemplo que el sonido no es un objeto de la luz. Y así como un aparato luminoso no puede iluminar el sonido, la voluntad no puede querer su fin. La voluntad quiere su libertad. Quiere querer, producir. Por eso no se trata de una incapacidad para lograr la identidad, sino de una apotencia o indifferentia (un no querer) ${ }^{49}$ respecto de la más alta identidad de los principios. Así, habla Schelling de un Gehemmtsein ${ }^{50}$. Apotencia quiere decir que el absoluto no persigue la alta identidad. La apotencia pone una dialéctica de revelación y ocultación donde no se busca ningún final; donde lo que se busca es sencillamente producir, y en este sentido revelar su infinitud por mediación de los productos y de la liberación de todo lo producido.

Parece además que si el querer quiere querer, esto debería ser entendido como querer un queriente, pues el queriente es además de querido, propiamente un querer. Por eso los productos no pueden ser comprendidos sólo como productos inertes. Debemos distinguir entre das mechanisch Produzierte y das organisch Produzierte, de modo que dentro de la Produktiosweise distingamos entre engendrar, erzeugen, y un mero poner, bewirken, en el que lo producido no es por sí, esto es, distinguir entre das organisch Hervorgebrachtes y das mechanisch Prodizierte de tal modo que se insufle de vida el spinozismo; y lo creado sea algo vivo y subsistente por sí mismo. O con otras palabras: lo producido debe ser a su vez productivo.

Tilliete sostiene que nos encontramos en la filosofía de Schelling une philosophie en devenir. Nos muestra la dificultad para afirmar conceptos permanentes. Sin embargo se puede habar a partir de 1809 de un concepto que va a perdurar. Aunque en 1809 aún no emplea la palabra "apotencia", creo que se puede decir que en la doctrina del Ungrund en las Philosophischen Untersuchungen se encuentra ya esta idea que permanecerá a lo largo del tiempo. Insisto, a pesar de que no se emplee aún dicha terminología en 1809. Y es que podemos encontrar en este año la siguiente consideración fundamental: la comparecencia de la determinabilidad del ser del absoluto y la determinación del absoluto nombran una relación de principios que puede ser caracterizada como aevum en tanto que modus durationum donde algo permanece oculto para siempre como Ungrund del proceso. Por eso domina lo finito, y sin embargo en lo finito se encuentra la huella del absoluto, de la unidad total de lo infinito y lo finito 51 .

puede leerse a su vez en: „Wenn man oder nicht begreift, dass das wahre Ende Freiheit ist, wird man kein Ende wollen und in grezenlose, ziellose Prozesse kommen. Aber nicht dort, sondern im Ende, im Télos ist Freiheit.“ Jacobs (2011b), p. 351s.

49 „Das Geheimnis nicht nur nicht wissen zu können, sondern es nicht wissen zu wollen, darin konkretisiert sich die Freiheit der Selbstständigkeit, welche zuletzt die Freiheit der Selbstbescheidung ist (...) Die Liebe wissen zu wollen, bedeutete daher ein Wissen ohne Liebe, welches Wissen, im Schellingschen Sinne, kein philo-sophisches Wissen wäre.“ Jürgensen (1997), p. 152.

50 „ein ursprüngliches Gehemmtsein der Produktivität.“ Einl., SW I/III, p. 287.

$51 \mathrm{PhK}, \mathrm{SW}$ I/V, p. 430. 


\section{El singular absoluto y lo oculto. Como conclusión de la primera parte de esta redacción}

La posterior doctrina de las potencias está constituida por la tríada „Sein-können“52, „Sein-müssen“53 y ,Sein-Sollen“. La primera potencia es el sujeto sin ser: reines können ${ }^{54}$ o reine Produktivität 55 . La segunda potencia, como objeto, es el producto. En esta tríada, el Sein-sollen es una llamada que apunta hacia lo que debiera ser: el equilibrio entre productividad y producto. Jacobs interpreta con razón: "al producto como objeto corresponde la productividad como sujeto, que no está pensado en el sentido de un sujeto consciente, sino como recoge el latín, en el sentido de subjectum, de lo que permanece debajo" 56 . La productividad se expresa en el producto: se revela y oculta al mismo tiempo en él. La productividad incondicional se oculta bajo la manifestación o producto a través de la cual se revela ${ }^{57}$. Ella puede mostrarse sólo en los productos, al tiempo que se oculta en ellos, porque ella nunca puede comparecer como un producto. Dice Jacobs: "la productividad incondicional no puede hacerse inmedatamente manifiesta" 58 . Pero ella es, y aquí está el punto de la cuestión, gracias a los productos, aunque este "gracias" bien entendido no designa una identificación, sino una Indifferenz de los principios. Y así como el pensar es pensar en lo pensado aunque no sea un pensado, la vida de Dios es en este sentido Eins von Allem ${ }^{59}$, y el mundo la revelación del Lebensprozesses de la personalización de Dios: la Bewusstwerdung en la naturaleza y en la historia60.

Siempre que el ser absoluto se proyecta en una forma determinada, sucede una inadecuación entre el ser (la productividad incondicional) y la finitud de la forma (productos). Pero no se trata de ninguna impotencia, porque sólo en virtud de esta apotencia el ser puede expresar su infinitud y determinabilidad. Por eso la indiferencia (Gleichgültigkeit) no nombra un no-poder, sino una apotencia o desinterés de las potencias que hace posible la expresión de la determinabilidad en tanto que dicha expresión no se logra por mediación de las potencias, sino que consiste en la

52 Cfr, GPPh, p. 133.

53 Cfr., GNPh, SW I/X, pp. 18-19.

54 „Die Freiheit des Anfangs ist reines Können und ist reiner, d.h. gleichgültiger Wille.“ Jantzen (1999), p. 60.

55 Krings (1985), p. 123.

56 Jacobs (2004), p. 75.

57 Cfr., VW, SW II, p. 382.

58 Jacobs (2004), p. 92.

59 „Doch bringt das nach Schelling eigentlich nicht eine Mehrzahl zustande, sondern es bleibt bei nur einem eizigen Subjekt, von dem alles gilt, was ausgesatgt wird.“ Buchheim (1992), p 172.

60 „Wir können nun zum voraus sagen, dass eigentlich der ganze Prozess der Weltschöpfung, der noch immerfort der Lebensprozess in der Natur und in der Geschichte - das dieser eigentlich nichts anderes als der Prozess der vollendeten Bewusstwerdung, der vollendeten Personalisierung Gottes ist." SPV VII, p. 433. 
constatación del absoluten Einzelwesen que se encuentra bajo toda determinación posible. De este modo queda dicho en qué sentido la apotencia designa la superioridad del ser singular.

Se trata en el fondo de la distinción entre posibilidad y realidad. Schelling quiere pensar eso que el ente es libre de todo lo sido, y de este modo el ser no en el sentido de una mera posibilidad. Por un lado la posibilidad se realiza, por otro ella es eso que el ente es, la causa de las causas o potencias. Causa de las causas quiere decir que lo $D a \beta$, el que, sale de sí. Las tres potencias existen sólo en la medida en que el absoluto singular o das absolute Einzelwesen (dicho de otro modo: eso que el ente es) es. No existe nada universal, nichts Allgemeines, ningún Was, tan sólo lo singular, lo Daß. Lo singular, das Einzelne, es lo real. Lo universal sólo lo posible. El ser singular es por consiguiente pensado como la condición de la posibilidad del ser universal, y tiene que permanecer por ello fuera del juego de las potencias.

Schelling concluye: la ,idea misma exige algo o una cosa de la que se pueda decir que es causa del ser (aition tou einai) y en este sentido lo que es, como algo tan sólo real, lo opuesto a lo universal, esto es lo singular, -que sin embargo y a través de la idea es determinado a la vez que se mantiene como cosa real independiente de la idea; de la que Kant habla sin que él pudiera alcanzarla"61. Lo singular62 es cada vez más el punto central de la filosofía tardía de Schelling. Cada vez más importante hasta que acaba por convertirse en el punto central de esta filosofía.

El ser es sólo reconocible a través del hecho del proceso. Al mismo tiempo permanece la apotencia como condición de posibilidad del proceso en la oscuridad del fundamento. Se trata de la posibilidad del proceso de la revelación a través de la expresión de la determinabilidad que se oculta tras cada manifestación o producto. Por razón de este ocultamiento puede tener lugar una liberación del ser respecto de todo lo finito. Así piensa Schelling un proceso real y vivo.

De las posibilidades, las tres potencias, somos pues conducidos a una realidad que es su causa 63 . Esta cuarta causa que es causa de las causas 64 no puede ser pensada por consiguiente por mediación de la doctrina de las potencias, sino como le Dieu caché fuera del procès cosmique ${ }^{65}$ : Dios permanece inexorablemente oculto y las tres potencias (atributos de Dios) son fundadas en su causa. El cuarto momento sólo puede llegar a nuestra atención si se percibe que no puede haber ninguna poten-

$61 \mathrm{DRPh}, \mathrm{SW}$ II/I, p. 292.

62 „Schelling deja de lado el concepto de Dios y parte del puro existente (blo $\beta$ Existierenden), en el que no es pensado nada más que el mero existir.“ Cruz (1993), p. 117.

63 „Es scheint also, daß wir zu einer vierten Ursache fortgehen müssen.“ DRPh, SW II/I, p. 399.

64 Cfr., ibid., p. 400.

65 "La liberté souveraine du Créateur est sauvagardée par la même processus de recel et de retrait. Dieu reste inexorablement le Dieu caché, le Potenzloser. Il se dissimule derrière les puissances qu'Il a distendues et extraverties, sa divinité comme actus purissimus demeure intangible, Il n'entre pas dans le procès cosmique." Tilliette (1987), p. 132. 
cia sin esta cuarta dimensión. De un lado este cuarto momento nos conduce hacia un límite del poder del conocimiento ${ }^{66}$, por otro lado nombra un originales Daß67. No eso que el ser es 68 , sino el ser de ese ser69.

\section{La influencia de Schelling en Heidegger}

\subsection{La Cuadratura}

No propondré buscar la influencia de Schelling en Heidegger allí donde en principio podríamos creer que deberíamos buscarla. Quiero decir, no en el escrito de 1936, sino, en su lugar, en el GA 54 (1942-43) que fue escrito tras el seminario de 1941. En esta edición encontramos una dialéctica de la desocultación y la ocultación que Heidegger aplica a su filosofía de la Cuadratura. Esta dialéctica dota a la filosofía del Geviert, que había sido formulada por primera vez en Der Rhein 70 (1935), de una nueva solidez. Tras 1935 nos encontramos con nuevas formulaciones de la misma: en Die Beiträge 71 (1936), Die Geschichte des Seyns (1938), Besinnung (1938/39), etc. Este proceso de evolución o desarrollo llega hasta 1949 (Das Ding) ${ }^{72}$. Ciertamente aún más tarde aparecen oscilaciones de la formulación del Geviert, pero no suficientemente significativas como para dejar de considerar la formulación de 1949 como la consolidación de la formulación madura. En este proceso de evolución se encuentra el texto de 1942/43 que había mencionado ocupando en el mismo un interesante e importante lugar, ya que es en él donde Heidegger consigue dar forma a lo que hasta el momento había sido tan sólo una llamada o invocación hacia una nueva época.

Heidegger había hablado en 1934 acerca de un nuevo camino y una nueva determinación para Alemania73. Esta llamada o invocación era una llamada hacia una nueva posibilidad histórica, hacia un nuevo inicio. Se trataba de una nueva época donde los hombres dejasen de creer en el poder de la voluntad para dominar la historia. Tal era el tema de la Cuadratura en su punto inicial. A partir de aquí se despliega una evolución de esta filosofía que desemboca en la siguiente tesis ontológi-

\footnotetext{
66 DRP, SW II/I, p. 413.

67 "God's original Daß.” Beach (1948), p. 141.

68 Cfr., DRPh, SW II/I, p. 412 y p. 385.

69 "It will be recalled that Schellings distinguisches between the was, or essential „wahtness" of things, and the Daß, or actualizing ,thahtness" of them.” Beach (1948), p. 136.

70 Geburt y Lichtstrahl, Noth y Zucht.

71 „Im selben Jahr, in dem Heidegger seine erste Vorlesung über die Freiheitsabhandlung hielt, also 1936, hatte er mit der Abfassung der Beiträge zur Philosophie begonnen." Friedrich (2009), p. 127.

72 Cfr., Mattei (2004). y Cfr., Stumpe (2002).

73 Cfr., Germanien, GA 39, p. 93.
} 
ca sobre el modo en el que el pensar es destinado: siempre que el fenómeno presente (Ergebnis) comparece, deja oculto tras de sí otras comparecencias posibles que permanecen en una suerte de estado oculto o Verborgensstand (Tierra) esperando el momento de la posibilidad de su Entbergung (Divinos). Los hombres (Mortales) buscan eso que fue ocultado y, de este modo, se elevan hacia el ámbito de lo desoculto (Cielo) ${ }^{74}$.

Esta estructura nuclear reposa sobre une dialectique de la révélation et du recel, que Heidegger resume en el GA 54 (1942/43) como sigue: "más bien es el Ent-bergen al mismo tiempo un Ent-bergen"75. Si traducimos Ent-bergen como desencubrir y Ent-bergen como resguardar-desocultando entramos en el ámbito de la Verborgen-Unverborgenheit, esto es, de lo oculto-desoculto, en el que hayamos un ocultar y un haber algo oculto al tiempo que un desocultar y un haber algo desoculto. La Cuadratura nombra así algo cuádruple, ein Vierfaches, que creo que podríamos resumir brevemente como sigue:

En primer lugar designa un ocultamiento, esto es, un encubrimiento que Heidegger expresa con distintos términos: Verhüllung, Verschleierung, Verdeckung, Aufbewahrung, Behütung ${ }^{76}$. Todos reunidos bajo la noción de Verborgenheit. En segundo lugar designa un ocultar, esto es, la acción de ocultar; aunque permanezca indeterminado quién o qué oculta ${ }^{77}$. El término que emplea Heidegger para este segundo sentido es el de Verbergen. En tercer lugar se puede hablar de un desocultamiento, en el sentido de una superación del ocultamiento 78 , y en virtud del cual debemos designar un cuarto momento: lo desoculto, Unverbergung. Aún tendría este desocultamiento un segundo sentido que no ha sido recogido y sobre el que Heidegger hace llamar la atención: que el ocultamiento no es permitido ${ }^{79}$. Esto llevaría a hablar no de una Unverbergung, sino de lo Unverborgene, de lo no ocultado.

Aunque Heidegger crea algo nuevo, podría haber aprendido de Schelling (especialmente en 1941) 80 que el ocultamiento es fundamental para salvaguardar la determinabilidad del ser (die Wesenheit). Heidegger dice en el seminario de 1942/43: la esencialidad de la verdad está en cada verdadero, de tal modo que sin lo verdadero no habría esencialidad ni verdad ${ }^{81}$. La pluralidad de sentidos de la ver-

\footnotetext{
74 Cfr., Rojas (2008).

75 Parmenides, GA 54, p. 198.

76 Cfr., ibid, p. 19.

77 Cfr., ibid, p. 19.

78 Ibid., p. 20.

79 Ibid, p. 20.
}

80, ,...) und das ist in der zweiten groß Vorlesung zu Schelling von 1941 wohl noch deutlicher -,dass Heidegger vor allem dem Blick auf Schellings Fundamentalunterscheidung von „dem Wesen, sofern es existiert und dem Wesen, sofern es bloß Grund von Existenz ist" richtet." Swenzfeuer (2010), p. 243.

81 „Die Aletheia ist das Wesen des Wahren: die Wahrheit. Diese west in allem Wesenden und ist das Wesen alles Wesens: die Wesenheit.“ Parmenides, GA 54, p. 242. 
dad nombra las figuras determinadas de la esencia de la verdad en la historia: certitudo, adaequatio, etc.82. Según la tesis de Heidegger en 1942/43, lo verdadero (das Wahre) es la forma que salvaguarda (wahren) la verdad (die Wahrheit) respecto de su pura esencialidad. Con otras palabras: la verdad se esencia en lo verdadero. En este proceso lo más significativo es que Heidegger busca mostrar la realidad de un ocultar fundamental. Sin un ocultamiento que pudiera ser considerado manifiestamente como lo ausente, no podría darse ningún movimiento en dirección al desocultamiento.

El ocultamiento no nombra una posible presencia, sino el fundamento de la posibilidad que sólo puede ser tal entre otras posibilidades. Esta pluralidad u horizonte de posibilidades es lo que funda la ausencia. Esta ausencia se oculta siempre. No puede ser ninguna presencia, sino que se trata del ser oscuro de las posibilidades que debe preceder a toda existencia y oposición.

Heidegger no dice que esta idea la haya tomado de Schelling. Él habla de Anaximandro y de Heráclito. Pero Heidegger habla de Heráclito en 1942 en el GA 54, y de Anaximandro en 1946 (Der Spruch des Anaximander), lo que da a pensar que se trata de un desarrollo posterior del seminario de 1942/43. Sea como sea Heidegger no se refiere en el GA 54 a Schelling. Y sin embargo, bien puede pensarse que dicho seminario no habría tenido lugar sin el seminario anterior sobre Schelling en 1941. Tanto que lo que Heidegger encuentra en las ideas de Anaximandro son las ideas que él habría aprendido a través de Schelling. Me refiero al Sich-Zurückziehen des absoluten Wesens ${ }^{83}$, que tras cada figura a través de la cual se manifiesta al mismo tiempo se oculta. Heidegger no habla por supuesto del Dios de Schelling, sino del Seinsgeschick, pero en éste se mantiene lo que en la noción de absoluto de Schelling es a mi juicio nuclear: la distinción entre determinabilidad y determinación.

Es difícil establecer la importancia que Schelling ejerce en Heidegger. Lo que sí podemos es decir que Heidegger se interesa por Anaximandro porque busca eso que no viene a la presencia, sino que se oculta. Y, por otro lado, que este juego de Geben y Entziehen lo podemos encontrar previamente en Schelling, al que Heidegger se había dedicado un año antes del GA 54 en el seminario de 1941, y cinco años antes del Dem Spruch des Anaximander.

\footnotetext{
82 „Im Wandel des Wesens der Wahrheit von der alétheia über die römische veritas zur mittelalterlichen adaequatio, rectitudo und iustitia und von hier zur neuzeitlichen certitudo, der Wahrheit als Gewissheit, Gültigkeit und Sicherheit wandelt sich mit das Wesen und die Art des Gegensatzes zwischen Wahrheit und Unwahrheit.“ ibid., p. 84.

83 „bei Schelling, innerhalb der deutschen Metaphysik, erhält das Begriffswort Existenz eine besondere Betonung, und zwar innerhalb der Unterscheidung: Grund und Existenz." Die Metaphisyk des deutschen Idealismus, GA 49, p. 196.
} 


\subsection{El ocultar. La cuadratura como ámbito del Verbergen-Unverborgenheit}

La filosofía moderna parte de la afirmación de que no se piensa lo que está ausente, y que las oscuridades metafísicas y trascendentales no son temas de teoría del conocimiento. De la mano del par presencia-ausencia adviene la siguiente pregunta rectora: ¿cómo viene a la presencia lo que está presente? ¿De dónde recibe la presencia su impulso presentificador? ${ }^{84}$ ¿Qué la empuja a salir de la ausencia? Husserl y Heidegger hablan sobre Vergegenwärtigung y Gegenwärtigen, mediante las cuales se nombra esta Erscheinen del fenómeno, sin la cual no habría nada que conocer, ningún Ergebnis 85 .

Sólo se puede hablar de lo presente. Por eso se habla de una Gegebenheit, porque la presencia tiene que ser dada. Y así nos topamos con la pregunta por la nada: ¿Por qué no es la nada? ¿Por qué el ser y no más bien la nada? Schelling y Heidegger piensan que no es sólo importante un dar, sino también un no-dar. Si el ocultar no existiera, tendríamos que hablar de una última presencia. Pero de lo que tenemos que hablar de hecho es de una sucesión de presencias. Y en este sentido Heidegger y Schelling nos dicen que si no se diera este ocultar, entonces no habría tampoco ninguna presencia ${ }^{86}$, ni ningún movimiento. No sólo pues no hay una última presencia, sino que no habría ninguna presencia en absoluto. Lo que Heidegger habría podido aprender de Schelling en 1941, por más que se refiera a Anaximandro en lugar de a Schelling, es la realidad del ocultar. Previamente a 1946 (Der Spruch des Anaximander), en el año 1942/43, esto es, después del seminario dedicado a Schelling, formula Heidegger por primera vez lo que será el corazón de la Cuadratura de ahora en adelante: la revelación de la determinabilidad por mediación de formas limitadas. Heidegger no quiere repetir la filosofía de la libertad de Schelling, pero sí recoge su pensamiento nuclear.

A partir de la página 195 habla Heidegger en el GA 54 de lo libre y lo abierto "en la amplitud de lo ilimitado y sin límite"87. Lo abierto nombra por un lado una determinabilidad, apeiron: "lo abierto y su extensión en la amplitud de lo ilimitado y sin límite es una zona previa en la que toda estancia se pierde en la inconsistencia"88. Por otro lado lo abierto nombra una determinación, péras: el rescate en el sentido de un desocultar que nombra al mismo tiempo un presenciar y un retirar89.

\footnotetext{
84 ,im Unterschied vom Augenblick als eigentlicher Gegenwart nennen wir je uneigentliche das Gegenwärtigen." Sein und Zeit, GA 2, p. 338.

85 Cfr., Husserliana 29, p. 193.

86 „Der Ort aber, worin die Kräfte (Potenzen) zur Scheidung kommen, ist das Nichts als Abgrund des in ihm zu erzeugenden und zu gebärenden Seins. Und das ist die Freiheit!“ Friedrich (2009), p. 77s.

87 Parmenides, GA 54, p. 214.

88 Ibid, p. 214.

${ }^{89}$ Cfr., ibid, p. 197.
} 
El propósito del seminario consiste en mostrar cómo lo abierto puede albergar la desocultación de las infinitas determinaciones. Lo abierto nombra una cuádruple dimensión de presencias y ausencias. No es ningún $d a$, sino la amplitud que es la determinabilidad de la Zuweisung des Seins.

Se trata de hecho de la idea de apeiron y su relación con lo determinado. Lo apeiron puede ser entendido sólo en relación a lo péras, porque lo apeiron no puede ser sin lo péras. Al mismo tiempo lo péras no es naturalmente lo apeiron, y por eso la sustitución y el cambio es la ley que aclara la polémica y necesaria unión ${ }^{90}$ entre apeiron y péras.

No se trata aquí sin embargo de un ciclo eterno in sensu Nietzsche, ya que Nietzsche habla contra el destino ${ }^{91}$. Tampoco la filosofía presocrática había pensado bien la finalidad. Heidegger, junto con Schelling, habla por el contrario acerca de la imposibilidad de una meta (eines Zieles) que no significa la imposibilidad de un fin, porque la finalidad de la revelación de la libre determinabilidad del ser infinito es posible por mediación de los productos. Encuentro aquí la cercanía entre la distinción de Form y Wesen en la filosofía de Schelling92 y la distinción entre Wahrheit y Wahre del seminario del 42/43. Distinción que más tarde se convertirá en la distinción entre Seinsgeschick y Gestalt des Grundes ${ }^{93}$. Aquí podríamos leer la propia confesión de Heidegger: "sin embargo es una vez más el ser que, siendo sólo él, se esencia haciendo ser" 94 . Esto significa: ambos pensadores piensan un ser que existe sólo él, y que se esencia haciendo ser. Naturalmente no es el Ser de Heidegger ni absoluto ni subjetividad incondicionada, pero permanece la misma concepción del principio de identidad.

\footnotetext{
90 „Krieg ist von allem der Vater, von allem König.“ DK 22 B53.

91 „Ich erlöste sie von der Knechtschaft unter dem Zwecke.“ Vor Sonnenaufgang, en: ASZ, NW I, p. 431.

92 Cfr., Jacobs (2011a), p. 89.

93 Cfr. Der Satz von Grund, GA 10, dreizehnte Stunde.

94 „Die Sache selbst (was diese Metaphysik zu denken hat) ist das Absolute. Weil dieses als unbedingte Subjektivität (d.h. Subjekt-Objekt), als Identität der Identität und Nichtidentität gedacht ist und die Subjektivität wesenhaft als willentliche Vernunft und damit als Bewegung, sieht es so aus, als decke sich das Absolute und seine Bewegtheit mit dem, was das seynsgeschichtliche Denken als das Ereignis erdenkt. Aber das Ereignis ist weder dasselbe wie das Absolute, noch ist es gar seine Entgegensetzung, etwa die Endlichkeit gegenüber der Unendlichkeit. Vielmehr ist im Ereignis das Seyn selbst als Seyn erfahren und nicht als ein Seiendes und schon gar nicht als das unbedingte Seiende und höchste Seiende gesetzt, trotzdem doch wieder das Seyn als dasjenige west, was allein ist.“ Heidegger (1971), p. 231.
} 


\section{Como conclusión de la segunda parte de esta redacción}

Heidegger y Schelling constituyen dos momentos de una filosofía de la libertad que tiene en su base una apotencia fundamental como fundamento de un proceso donde se hace manifiesta la determinabilidad del Wesen, y donde al mismo tiempo, éste, se oculta tras las distintas Erscheinungen. En pocas palabras lo que leemos en esta filosofía es una defensa de una nueva idea de libertad que se opone a la herrschende Erscheinung. Libertad quiere decir apertura a nuevas posibilidades que se ocultan tras cada aparición. Si no existiera esta ocultación, nos encontraríamos entonces con una aparición dominante y necesaria que sería la única posibilidad, y de este modo nos toparíamos con el fin de la determinabilidad.

En contraposición a la unidad absoluta que tendría como consecuencia la disolución de los principios, la apotencia nombra en la filosofía de Schelling, como se dijo, una indiferencia respecto de la identidad de los principios. Por este motivo sucede una "afirmación del tiempo"95 donde acontece la unión de los principios en un equilibrio producido por el misterioso amor. No se trata pues de una impotencia, sino del hecho de que para que sea posible una liberación respecto de las formas finitas, tiene que existir un desinterés respecto de cualquier determinación, también de la más alta determinación. Por eso la posibilidad de la revelación del ser absoluto sólo es posible en el tiempo. Porque esta revelación sólo es posible en la medida en que la determinabilidad no pretende su identificación con ninguna determinación. Ella toma distancia con respecto a la identidad para amar el proceso y la vida. La unión no crea pues ninguna indistinción de los principios, porque aunque no es pensable un destino indeterminado, eso no significa que tengamos que pensar una determinación final. La voluntad libre es libre por razón de una ausencia que funda un nuevo horizonte de determinaciones, en función de los cuales estamos en condiciones de hablar de una Be-freiung de las formas limitadas.

Heidegger se interesa por la ocultación del ser tras las determinaciones. Piensa que la ocultación crea la posibilidad de nuevos modos y tipos en los que ver bajo el claro aquello que se manifiesta como ausente. Heidegger no quiere repetir la Freiheitsphilosophie de Schelling, pero recoge el pensamiento nuclear de la apotencia schellingiana. Schelling y Heidegger encuentran ambos en el fondo una apotencia sin la cual no sería posible ninguna determinación más, lo que significaría el fin de la productividad o determinabilidad. Esta apotencia nombra el fundamento como indifferentia (no querer saber) respecto de la identidad entre Bestimmbarkeit y Bestimmung: si no se diera esta apotencia no se podría hablar de movimiento, cambio ni vida. Esta indiferencia mantiene viva la distinción de los principios, su indiferenciación o Indifferenz. El destino del ser del que habla Heidegger en relación a

95 „Daher schließlich Freiheit als Entschluß zum Unausweichlichen (Bejahrung der Zeit).“ ibid., p. 232. 
las figuras de la determinación del fundamento o Schickung prosigue la doctrina de las potencias de Schelling en este sentido y por eso puedo decir que Heidegger es el eslabón que engancha la filosofía de Schelling a la historia de la filosofía después de que pareciera que ésta se había olvidado de él para tomar un curso distinto96.

\section{Abreviaturas}

$\begin{array}{ll}\text { ASZ } & \text { Also sprach Zarathustra } \\ \text { DRPh } & \begin{array}{l}\text { Darstellung der reinraionalen Philosophie = Philosophische } \\ \text { Einleitung in die Philosophie der Mythologie }\end{array} \\ \text { Einl. } & \text { Einleitung zu dem Entwurf eines Systems der Naturphilosophie } \\ \text { EV } & \text { Erlanger Vorträge } \\ \text { GA } & \text { Gesamtausgabe } \\ \text { GNPh } & \text { Zur Geschichte der neueren Philosophie } \\ \text { GPPh } & \text { Grundlegung der positiven Philosophie. Münchner Vorlesung } \\ \text { NW } & \text { Friedrich Nietzsche Werke in zwei Bänden } \\ \text { PhK } & \text { Philosophie der Kunst } \\ \text { PhU } & \text { Philosophische Untersuchungen über das Wesen der } \\ & \text { menschlichen Freiheit und die damit zusammenhängenden } \\ & \text { Gegenstände } \\ \text { SPV } & \text { Stuttgarter Privatvorlesungen } \\ \text { SW } & \text { Sämmtliche Werke } \\ \text { WA } & \text { Die Welttaler } \\ \text { VW } & \text { Von der Weltseele }\end{array}$

\section{Referencias bibliográficas}

ARIStoteles (1936): Aristotle’s physics, Ross (Ed.), Oxford University Press, New York.

BeACH, E. A. (1948): The Potencies of God(s), State University of New York Press, New York.

BuchneIM, T. (1992): Eins von Allem, Die Selbstbescheidung des Idealismus in Schellings Spätphilosophie, Meiner, Hamburg.

Cruz, J. (1993): Ontología de la razón en el último Schelling. Acerca de la introducción a la filosofía de la revelación, Universidad de Navarra, Pamplona.

\footnotetext{
96 "Es muy posible que el camino definido por la filosofía de Schelling siga una dirección por la que no continuó la propia historia de la filosofía y que en consecuencia señalaría un trayecto personal de Schelling, único transeúnte, perdido en un laberinto producido por los sueños de una razón visionaria, metafísica, especulativa y mística.” Leyte (1999), p. 13.
} 
Diels, H. (1930): Die Fragmente der Vorsokratiker, Weidmann, Berlin.

DuQue, F. (1998a): Historia de la Filosofia Moderna. La era crítica, Akal, Madrid. DuQue, F. (1998b): "La puesta en libertad de la filosofía. El concepto de libertad y la libertad del concepto en Schelling", en: Daimon 16, pp. 41-56.

FALgueras, I. (1976): La res cogitans en Espinosa, EUNSA, Pamplona.

FALGueras, I. (1999): "El inicio del método de las potencias", en: Leyte A. (Ed.), Una mirada a la filosofía de Schelling, Universidad de Vigo, Vigo, pp. 247-256. FrIEDRICH, H-J. (2009): Der Urgrund der Freiheit im Denken von Böhme, Schelling und Heidegger, Frommann-Holzboog, Stuttgart-Bad Cannstatt.

GarcíA, J. A. (1999): "Siempre lo mismo o algo nuevo", en: Thémata 22, pp. 119131.

GarcíA, J. A. (2007): „La naturaleza según Nicolás de Cusa (1401-1464)“, en: Espiritu 131, pp. 5-23.

HeIDEGGER, M. (1927): Sein und Zeit, GA 2, Klostermann, Frankfurt a.M..

HeidegGer, M. (1971): „Ausgewählte Stucke aus dem Manuskripten zur Vorbereitung des Schelling-Seminars S.S. 1941“, en: Feick, H. (Ed.), Schelling Abhandlung über das Wesen der menschlichen Freiheit (1809), Niemayer, Tübingen, pp. 201-236.

HeIDEgGer, M. (1992): Parmenides, GA 54, Klostermann, Frankfurt a.M..

HeIDEgGER, M. (1995): Logik. Die Frage nach der Wahrheit, GA 21, Klostermann, Frankfurt a.M..

HeIDEGger, M. (1997): Der Satz vom Grund, GA 10, Klostermann, Frankfurt a.M.. HeidegGer, M. (1999): Hölderlins Hymnen Germanien und Der Rhein, GA 39, Kolstermann, Frankfurt a.M..

HeidegGer, M.(2006): Die Metaphysik des deutschen Idealismus. Zur erneuten Auslegung von Schelling: Philosophische Untersuchungen über das Wesen der menschlichen Freiheit und die damit zusammenhängenden Gegenstände (1809), GA 49, Klostermann, Frankfurt a.M..

Halfwassen, J. (2010): „Freiheit als Transzendenz. Schelling Bestimmung der absoluten Freiheit in den Weltaltern und in der Philosophie der Offenbarung", en: Hühn-Jatzen (Ed.), Heideggers Schelling-Seminar (1927/28), FrommannnHolzboog, Stuttgart-Bad Canstatt.

Heimsoeth, H. (1958): Die Sechs großen Themen der Abendländischen Metaphysik, Kohlhammer, Darmstadt.

Hennigfeld, J. (2001): Friederich Wilhelm Joseph Schellings >Philosophische Untersuchungen über das Wesen der menschlichen Freiheit und die damit zusammenhängenden Gegenstande, Wissenschaftliche Buchgesellschaft, Darmstadt.

HuSSERL, E. (1993): Die Krisis der europäischen Wissenschaften und die transzendentale Phänomenologie, Husserliana 29, Kluwer, Dordrecht. 
HutTER, A. (1996): Geschichtliche Vernunft, Suhrkamp, Frankfurt a.M..

JACOBS, W.G. (1999): „Das Weltbild der modernen Naturwissenschaften und die

Schellingsche Naturphilosophie“, en: Fehér-Jacobs (Ed.), Zeit und Freiheit, Kétef, Budapest, pp. 37-54.

JACOBS, W.G. (2011a): „Natur in der Kunst“, en: Danz-Jantzen (Ed.), Gott, Natur, Kunst und Geschichte, Vienna University Press, Göttingen, pp. 85-102.

JACOBS, W.G. (2011b): „Philebos - der Kern platonischer Weisheit. Zum Problem der Endlichkeit in Schellings Philosophie“, en: Galland-Saymkowiak (Ed.), Das Problem der Endlichkeit in der Philosophie Schellings, Lit, Zürich/Berlin, pp. 333-352.

JaCOBS, W.G. (2004): Schelling lesen, Frommann-Holzboog, Stuttgart-Bad Cannstadt.

JantZen, J. (1999): „Jenes Übertreten des Subjekts ins Objet“, en: Leyte, A., Una mirada a la filosofia de Schelling, Universidad de Vigo, Vigo, pp. 39-64.

JÜRgENSEN, S. (1997): Freiheit in den Systemen Hegels und Schellings, Königshausen und Neumann, Würzburg.

KRINGS, H. (1985): „Natur als Subjekt. Ein Grundzug der spekulativen Ohysik Schellings, in: Natur und Subjektivität. Zur Auseinandersetzung mit der Naturphilosophie den jungen Schelling“, en: Heckmann-Krings-Meyer (Ed.), Referate, Voten und Protokolle der II. Internationalen Schelling-Tagung Zürich 1983, Meyer, Stuttgart-Bad Cannstatt, pp. 111-128.

Koloswki, P. (2001): Philosophie der Offenbarung, Schöningh, Paderborn.

LeYte, A. (1999): „Épocas de la razón: lógica e historia en Schelling“, en: Leyte, A., Una mirada a la filosofía de Schelling, Universidad de Vigo, Vigo, pp. 1324.

LeYTE, A. (2010): „Zeit-Denken. Zu einem nicht-begrifflichen Zugang zur Zeit bei Schelling und Heidegger", en: Hühn-Jatzen (Ed.), Heideggers SchellingSeminar (1927/28), Frommannn-Holzboog, Stuttgart-Bad Canstatt, pp. 139162.

MatteI, J-F. (2004): Heidegger. L'enigme de l'être, Press Universitaires, Paris, 2004.

Nietzsche, F. (1930): Friedrich Nietzsche Werke in zwei Bänden, I, Kröner, Leipzig.

Plato (1838): Opera omnia, Hennings, Gothae.

ROJAS, A. (2008): La Cuadratura. La última palabra del pensamiento ontológico de Heidegger, Universidad de Málaga, Málaga.

Schelling, F.W. J. (1856): Sämmtliche Werke, II/I, Cotta, Stuttgart-Ausburg.

Schelling, F.W. J. (1857): Sämmtliche Werke, I/II, Cotta, Stuttgart-Ausburg.

Schelling, F.W. J. (1858): Sämmtliche Werke, I/III, Cotta, Stuttgart-Ausburg.

Schelling, F.W. J. (1859): Sämmtliche Werke, I/V, Cotta, Stuttgart-Ausburg. 
Schelling, F.W. J. (1860): Sämmtliche Werke, I/VII, Cotta, Stuttgart-Ausburg.

Schelling, F.W. J. (1861): Sämmtliche Werke, I/VIII, Cotta, Stuttgart-Ausburg.

SCHELling, F.W. J. (1861): Sämmtliche Werke, I/IX, Cotta, Stuttgart-Ausburg.

Schelling, F.W. J. (1861): Sämmtliche Werke, I/X, Cotta, Stuttgart-Ausburg.

SChelling, F.W. J. (1972): Grundlegung der positiven Philosophie. Münchner Vorlesung, Fuhrmans (Ed.), Bottega D’Erasmo, Turin.

Schelling, F.W. J. (1994): Timaeus (1794), Frommann-Holzbog, Stuttgart-Bad Canstatt.

Stumpe M. (2002): Geviert, Gestellt, Geflecht. Die logische Strukur späten Texten, BoD, Braunschweig.

Sturma, D. (1995): „Präreflexive Freiheit“, en: Höffe O. y Pieper A. (Ed.), Über das Wesen der menschlichen Freiheit, Akademie Verlag, Berlin, pp. 149-172.

SwENZFEUER, S. (2010): „Natur und Sein. Affinitäten zwischen Schelling und Heidegger“, en: Hühn-Jatzen (Ed.), Heideggers Schelling-Seminar (1927/28), Frommannn-Holzboog, Stuttgart-Bad Canstatt, pp. 227-261.

Tilliete, X. (1987) : L'Absolu et la philosophie, PUF, Paris.

TILLIETE, X. (1992): Schelling. Une philosophie en devenir, II: La dernière philosophie 1821-1854, Vrin, París.

Alejandro Rojas Jiménez

Bayerische Akademie der Wissenschaften

Kommission zur Herausgabe der Schriften von Schelling

rojasj_a@yahoo.es 